\title{
Open-Source Programming of Cardiovascular Pressure-Flow Dynamics Using SimPower Toolbox in Matlab and Simulink
}

\author{
Ofer Barnea*
}

Department of Biomedical Engineering Tel Aviv University Ramat Aviv 69978 Israel

\begin{abstract}
A toolbox for simulation of the cardiovascular system has been constructed for use in Matlab Simulink (The MathWorks Inc.). The toolbox contains basic elements such as vessel segments, cardiac chambers, valves and shunts that can be graphically arranged and connected to directly simulate the circulation with the desired level of detail. The toolbox is based on the SimPower toolbox to allow simple connections between the different elements. One difficulty that remains is the need to know the values of the various parameters. The toolbox is an open-source script that is available for download. It is expected that researchers that will use the toolbox will refine it, enhance its capabilities and share their code with others.
\end{abstract}

Keywords: Cardiovascular system, mathematical models.

\section{INTRODUCTION}

The cardiovascular system (CVS), the heart, the blood vessels and the blood flowing in the system, received a considerable amount of attention from scientists of various fields including physiologists, biologists, engineers and physicists. Models arising from many different approaches have been developed to elucidate mechanisms of the normal and pathological function and structure of the system. Models and computer simulations were also designed to study specific effects and interactions of the CVS with physiological as well as man-made systems. These models help to understand and demonstrate behavior of the system's various aspects as well as help in eliminating or minimizing the need for animal experiments.

Mathematical models may be subdivided into two major categories: a) mathematical descriptions of observed phenomena or interactions and b) mathematical descriptions of physiological or physical mechanisms or structures. The first category includes relations such as polynomials or exponential functions that describe the effect of one variable on others based on averages of observed values. Coefficients and constants in the mathematical description are not necessarily related to physical or physiological parameters. In second category, the one is described in this paper, coefficients and constants are related to physical or physiological parameters of the modeled system or organ. These models are based on physical/physiological behavior of the system.

Pressure and flow relations in the cardiovascular system would ideally retain all the terms in the Navier-Stokes equations and an expression describing elastic wall motion. Finite-element methods are used to compute the flow and

*Address correspondence to this author at the Department of Biomedical Engineering Tel Aviv University Ramat Aviv 69978 Israel;

Tel: +972-3-6408658; Fax: +972-3-6405830;

E-mail: barneao@eng.tau.ac.il pressure with high accuracy.However, the mathematical and computational complexity is very large and often unnecessary. On the other hand, lumped parameter models can describe pressure flow phenomena without providing such exact solution but do provide reasonable pressure-flow relations and allow the study of a system behavior.

The heart can be represented by finite element models where the dynamics of each segment of tissue is calculated $[1,2]$. In contrast, when exact regional details are not required, a lumped-parameters model can represent the function of the whole contracting heart and provide reasonable predictions of volume, pressure and flow [3].

\section{LUMPED PARAMETERS MODELS}

For simulating blood vessels, a balance between accuracy of the finite element solutions and the lumped parameter models can be achieved by assuming that the vessels can be divided into segments, each with lumped values of hemodynamic parameters. Noordergraaf and co-workers constructed an analog model for the human systemic arterial tree composed of 113 segments, each about $5 \mathrm{~cm}$ long [4]. By varying elements of the model, they were able to study hemodynamic changes in the system and to predict its response to various inputs. A major contribution to lumped parameter multi-element models was published in 1967 by Rideout and Dick [5]. Following an assumption that the second partial derivative of the velocity with respect to $x$ (along the vessel) is of negligible value, they arrived at the following linearized equations:

$$
\begin{aligned}
& \frac{\partial P}{\partial x}=\rho \frac{\partial w}{\partial t}-\eta\left(\frac{\partial^{2} w}{\partial r^{2}}+\frac{1}{r} \frac{\partial w}{\partial r}\right) \\
& \frac{\partial P}{\partial r}=\rho \frac{\partial u}{\partial t}-\eta\left(\frac{\partial^{2} u}{\partial r^{2}}+\frac{1}{r} \frac{\partial u}{\partial r}-\frac{u}{r^{2}}\right)
\end{aligned}
$$


(where $P$ is pressure and $w$ and $v$ are velocities in the $x$ and $r$ directions, respectively). With the equation of continuity that expressed the incompressibility of blood (in cylindrical coordinates):

$$
\frac{\partial u}{\partial r}+\frac{u}{r}+\frac{\partial w}{\partial x}=0
$$

Integration was performed along the radius, pressure was assumed constant in the whole cross section area and mean velocity was replaced by volumetric flow. Thus, eq. 1 was converted to a discrete form shown by eq. 4

$$
P_{n}-P_{n+1}=L_{n} \frac{d Q_{n}}{d t}+R_{n} Q_{n}
$$

Where $\mathrm{P}_{\mathrm{n}}$ is the pressure at the n'th segment, $Q_{n}$ is volumetric flow, $L_{n}$ represent inertia and $R_{n}$ viscosity.

This model was further developed in two ways. Vessel compliance was added and a non-parabolic velocity profile was considered to calculate $R_{n}$ and $L_{n}$ in eq. 4 . A capacitor representing vessel compliance neglected before was added to the model as shown in eq. 5 :

$Q_{n}-Q_{n+1}=C \frac{d P_{n}}{d t}$

This satisfies the continuity equation regarding longitudinal and radial flow components. Equations 4 and 5 describe the pressure-flow relations in a vessel segment. Elements such as resistor and capacitor were also used to represent viscoelastic behavior of the vessel wall such as Voigt and Maxwell models. Barnea et al. [6] described hybrid models that use also experimental data for the vessel segment pressure-volume relations in the form of a polynomial along with a series resistor and inertance to obtain more accurate nonlinear effects at different pressures. This model also enabled instantaneous update of vessel radius with instantaneous pressure and volume changes.

A model of the ventricle is expected to be independent of both preload and afterload and is supposed to predict the ventricular contraction and ejection pattern for various preload and afterload conditions. The suga-sagawa widely accepted "varying-elastance" model achieves most of these goals. The model is based on Otto Frank's work. He represented the frog's cardiac function in the pressurevolume plane more than one hundred years ago, laying the foundation for today's most widely accepted "varying elastance" ventricular model. Suga $[3,7,8]$ further developed that model and established the load independence of the end systolic pressure-volume relationship of the heart [9, 10]. This made the concept very attractive for modeling since cardiac function can be described independently of the filling pressure and aortic load. When this ventricular model is coupled to a model of the circulation, one can predict the behavior of the heart in response to various perturbations. The ventricular "elastance" was defined:

$$
E(t)=\frac{P_{L V}(t)}{V_{L V}(t)-V_{0}}
$$

where $P_{L V}(t)$ and $V_{L V}(t)$ are the left ventricular instantaneous pressure and volume respectively, and $V_{0}$ is the volume axis intercept of the line connecting the end systolic pressurevolume points for differently loaded beats.

The model was further supported by the demonstration of the straight regression line of isochronal pressure-volume ratios for differently loaded beats at steady inotropic state [11]. End systolic pressure-volume relationship was widely accepted as an index of cardiac contractility and has been named ESPVR (end systolic pressure volume relationship). ESPVR is often used interchangeably with $E_{\max }$ which is the maximum value of $E(t)$ - not necessarily at end systole.

In order to describe instantaneous left ventricular pressure, equation 6 may be rewritten as follows:

$$
P_{L V}(t)=E(t)\left[V_{L V}(t)-V_{0}\right]
$$

This relationship approximates the cardiac function for small stroke volumes, but does not predict correct pressure flow relationships for larger stroke volumes and it is also somewhat affected by loading conditions. In the late 1970's several works demonstrated the weak point of this model. Suga and Yamakoshi and Suga and colleagues presented P-V trajectories where for the same end systolic volume, the end systolic pressure of an isovolumic beat was higher than the end systolic pressure of an ejecting beat [12, 13]. This pressure deficit was observed by others and there were several attempts to improve the model. Campbell and his colleagues added a series resistor to the electrical analog model of the heart, which was based on the varying elastance model $[14,15]$. The role of the resistor was to account for flow related pressure drop. Shroff et al. demonstrated a close fit of pressure and flow patterns generated by a model where the series resistor is proportional to instantaneous left ventricular pressure and experimental data $[16,17]$.

\section{THE TOOLBOX}

To model the hemodynamic aspects of the cardiovascular system, we need a few elements. We need a model of a contracting chamber, a model of a valve, and a model of a blood vessel. All models should be able to represent nonlinear aspects of the physical properties of the organs as well as pathologies. Also choices must be made since several different models are available.

The heart model was based on the "varying elastance". Pressure-volume relations are used to describe both the contracting phase (eq 8) and the diastolic phase (eq 11). Systolic contraction is based on a normalized elasticity function $E_{N}$

$$
P(t)=E_{\max } E_{N}\left(t / t_{\max }\right) \cdot\left(V(t)-V_{0}\right)
$$

Where $P$ is the chamber's pressure, $E_{\text {max }}$ is the maximal chamber pressure-volume relation, $V(t)$ is instantaneous chamber volume and $V_{0}$ is a constant. $t_{\max }$ is the time when the elastance function assumes its maximum value normally close to end systole and may be adjusted to correct for changes in systolic duration at different heart rates.

Left ventricular $\mathrm{E}_{\mathrm{N}}\left(\mathrm{t}_{\mathrm{n}}\right)\left(t_{n}=t / t_{\max }\right)$ function is described in Eq. 9 while Eq 10 describes the RV, RA and LA $E_{N}$ function. Note, only the positive values are used.

$$
E_{N}\left(t_{n}\right)=-1.841 t_{n}^{3}+2.685 t_{n}^{2}+0.158 t_{n}
$$


$E_{N}\left(t_{n}\right)=-3.374 t_{n}{ }^{3}+6.568 t_{n}{ }^{2}-1.934 t_{n}$

The diastolic passive stage is related to the blood filling stage of the chamber is characterized by a simplified pressure-volume relation:

$$
P(t)=e^{(K \cdot V(t))}-1
$$

The implementation under the SimPower toolbox is shown in Fig. (1). Current is analogous to blood flow, voltage is analogous to pressure, and charge to blood volume. This special implementation allows the use of Simulink where blocks are simply attached to other blocks, thereby transferring pressure and flow data.

In a cardiac chamber input flow is measured and integrated to obtain instantaneous value of chamber blood volume. Using eq. 8 with a polynomial (eqs. 9, 10 or 11 depending on the case), we obtain the chamber pressure. The charge output indicates blood volume.

The user may control all cardiac chamber parameters. Reduced contractility can simulate global ischemia, increased diastolic compliance can simulate diastolic dysfunction, and increased $V_{0}$ shifts the PV loop to the right to simulate local ischemia.

The valve is described by a versatile model that accounts for stenosis and insufficiency as well as for leakage and reverse flow. It also includes the dynamic pressure-volume relations of the leaflets represented by a vsicoelastic model (resistor and capacitor). The diodes represent the ideal valve but the software allows the user to alter forward resistance, backward resistance and opening pressure as shown in the right panel of Fig. (2).
Blood vessels may be modeled using the basic segment model shown in the left panel of Fig. (3). This model can be used either as a lumped parameter representation of the whole vascular load (windkessel model) or as a very short segment in a cascaded structure (shown in the right panel of Fig. 3). For example the aorta can be modeled as a multi-segment vessel with different parameter values at each segment if information along the aorta is required and the user wishes to emphasize the effects of delay and reflected waves. To assist in building the model "Y" type vessel connections are also provided. The same model can be used for any vessel since the parameter values are preset by the user. In the case of a vessel that is exposed to external pressure, like a coronary artery, an external controlled pressure source can be added.

The SimPower implementation is shown in Fig. (4). It includes preset resistor, inductor (for intertance) and a nonlinear capacitor that represents the nonlinear compliance using a polynomial.

The toolbox was written using Matlab version 7 with Simulink and the SimPower toolbox. This configuration allows the user to simply connect and element to the other and run the program. Several examples were previously publishedi.

As an open source code, the whole toolbox can be downloaded at the address http://www.eng.tau.ac.il/ barneao CVT.ZIP. The package includes help files and installation instructions. Due to changes in newer versions of the Matlab application, the toolbox is not compatible. It is recommended to use it under version 7.

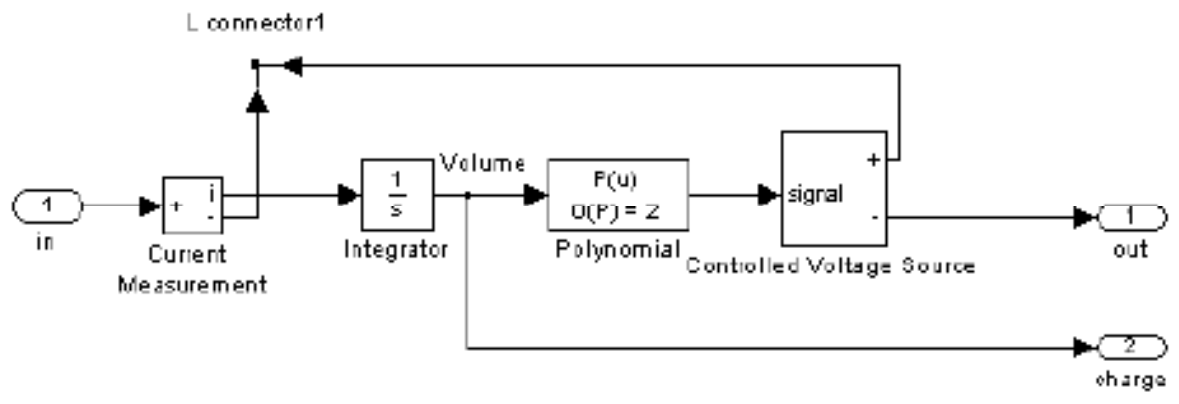

Fig. (1). Implementation of a cardiac chamber.
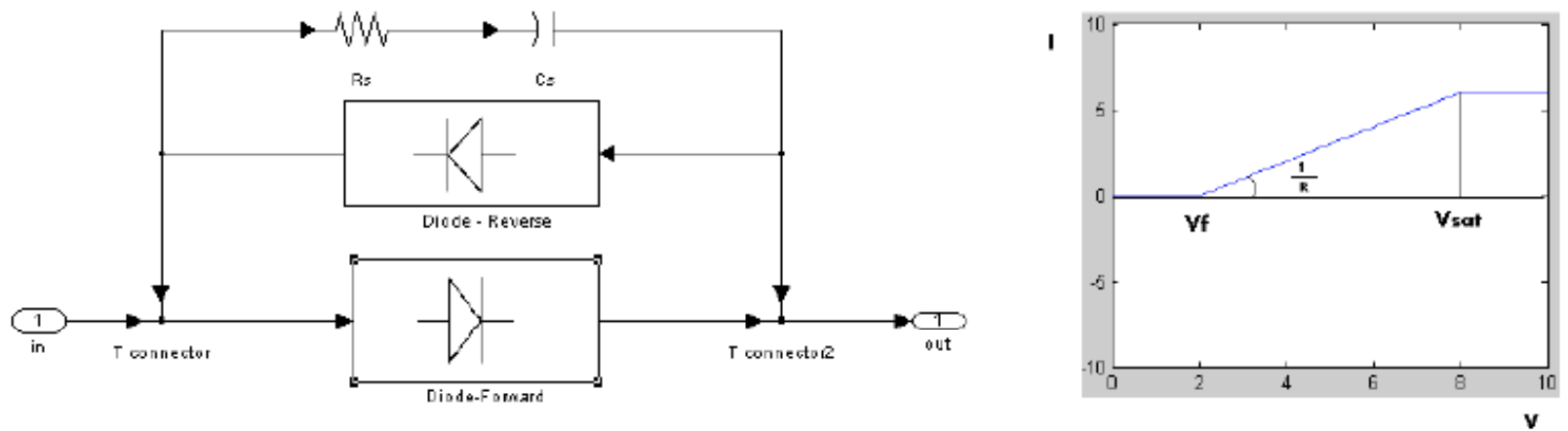

Fig. (2). Left panel: Valve model implementation that includes viscoelstic elements and forward and reverse valves with variable resistances. Right panel: diode characteristics and parameters where voltage is analogous to pressure and current to flow. 


\section{AN EXAMPLE}

An example is shown in Fig. (5) to demonstrate the toolbox. A varying elastance ventricle model is coupled to a three element windkessel model via a valve. The ventricle is filled by a reservoir of fixed pressure via a valve. In addition, there are pressure and flow monitors that present waveforms. Fig. (6) shows sample waveforms for this model. More elaborate models are presented in Sheffer et al. [18].
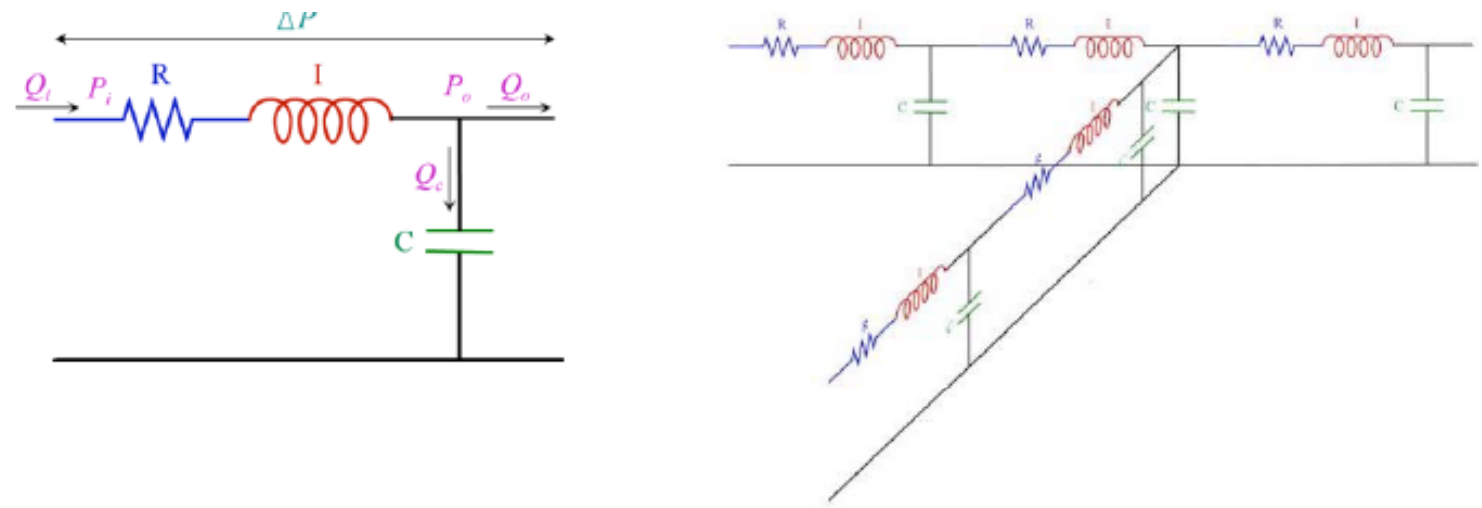

Fig. (3). Electrical analog models of flow in blood vessels.

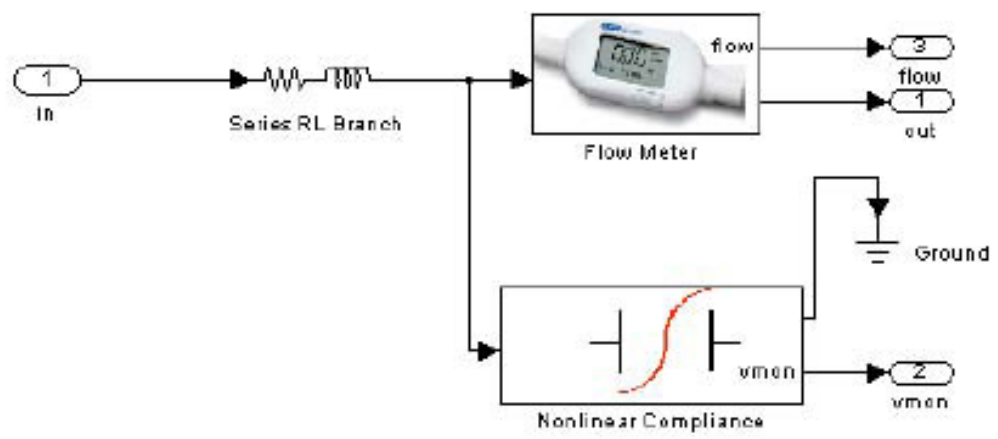

Fig. (4). Implementation of a vessel segment.

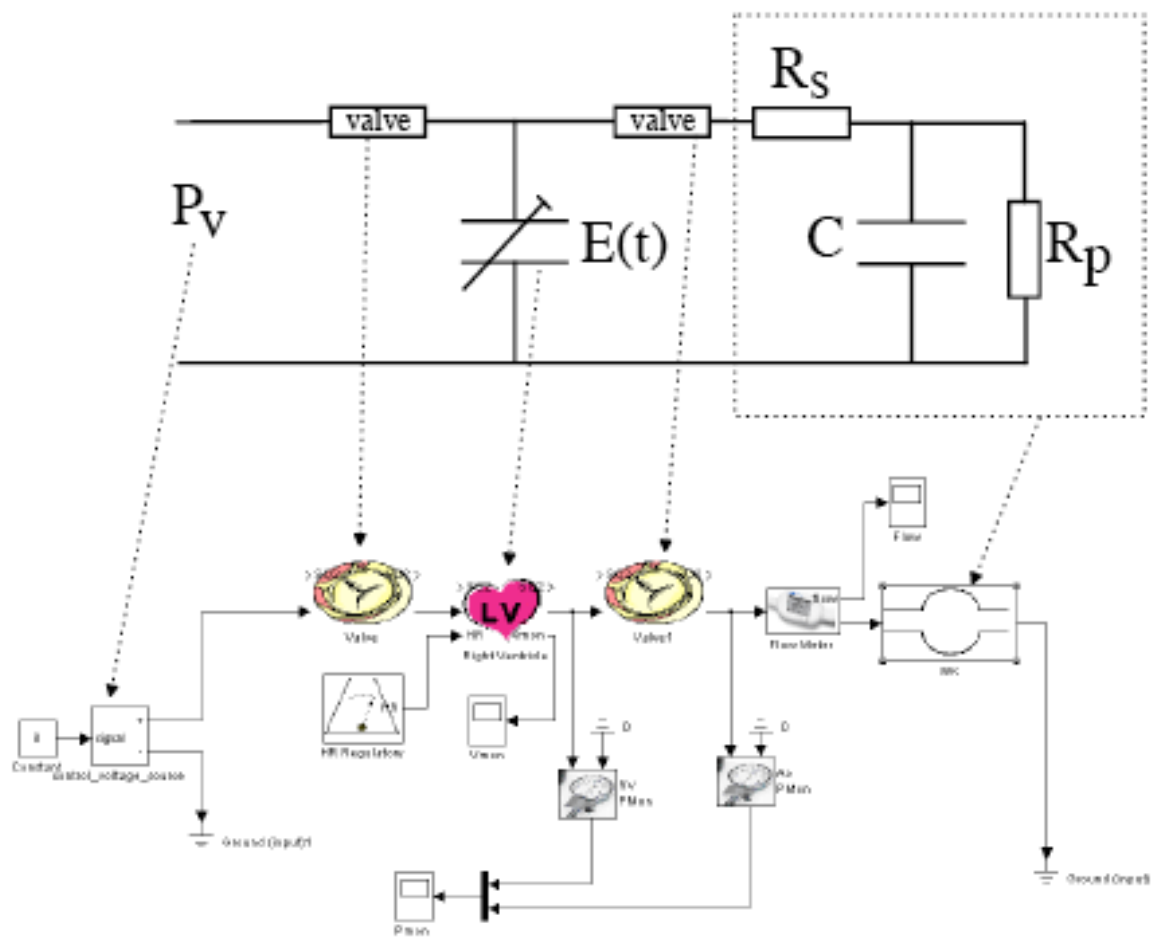

Fig. (5). An example of model construction using the toolbox. 


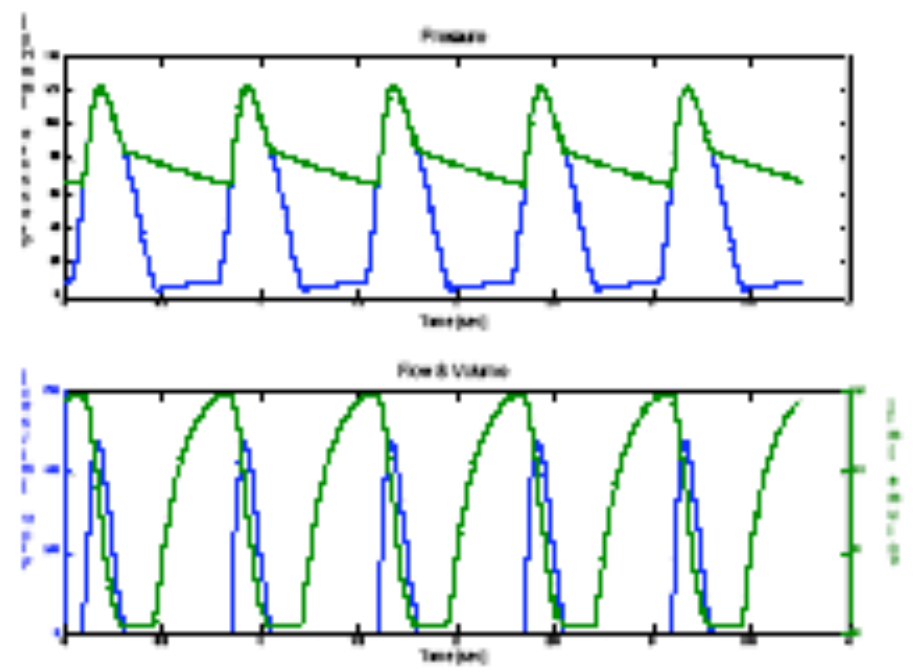

Fig. (6). Ventricular blood volume, pressure and flow computed by the toolbox for the model shown in Fig. (5).

\section{DISCUSSION}

The toolbox offers easy construction of cardiovascular models and runs well on Matlab version 7. The use of the SimPower toolbox allows easy connections between elements of the system once the analog circuit has been determined including nonlinearities represented by polynomials. However, one difficulty, as in any model, is the selection of parameters' values. In this version the parameters must be properly set. Thus, to use the toolbox, prior knowledge regarding the physical aspects of the system is required. In future versions of the toolbox we intend to change the type of parameters. Instead of setting a resistance value or an inertance value in a vessel model it could be more user friendly to set its diameter, length, vessel wall elastic factor and blood viscosity or hematocrit. In addition, users can develop models of various organs and share them with other users.

\section{REFERENCES}

[1] Hunter PJ, Pullan AJ, Smaill BH. Modelling Total Heart Function. Annu Rev Biomed Eng 2003; 5: 147-77.

[2] Guccione JM, Costa KD, McCulloch AD. Finite element stress analysis of left ventricular mechanics in the beating dog heart . J Biomech 1995; 28: 1167-77.

[3] Suga H. Left ventricular pressure volume ratio in systole as an index inotropism. Jpn Heart J 1971; 12: 153.

[4] Noordergraaf A. Analog of the Arterial Bed. In: Attinger EO, Ed. International Symposium on Pulsatile Blood Flow, New York: McGraw Hill 1964.

[5] Rideout VC, Dick DE. Difference-differential equations for fluid flow in distensible tubes. IEEE Trans Biomed Eng 1967; 14: 171.

[6] Barnea O, Moore TW, Jaron D. Cardiac energy considerations during intra-aortic balloon pumping. IEEE Trans Biomed Eng 1990; 37(2): 170-81.
[7] Suga H. Time course of left ventricular pressure-volume relationship under various end-diastolic volume. Jap Heart J 1969; 10: 509 .

[8] Suga H. Time course of left ventricular pressure-volume relationship under various extents of aortic occlusion. Jap Heart J 1970; 11: 373.

[9] Suga H, Sagawa K, Shoukas A. A Load independence of the instantaneous pressure-volume ratio of the canine left ventricle and effects of epinephrine and heart rate on the ratio. Circ Res 1973; 32: 314 .

[10] Suga H, Sagawa K. Instantaneous pressure-volume relationship and their ratio in the excised, supported canine left ventricle. Circ Res 1974; 35: 117.

[11] Sagawa K, Suga H, Shoukas A, Bakalar KM. End systolic pressure/volume ratio: a new index of ventricular contractility. Am J Cardiol 1977; 40: 748-53.

[12] Suga H, Yamakoshi K. Effects of stroke volume and velocity of ejection on end-systolic pressure of canine left ventricle. Circ Res 1977; 40: 445-50.

[13] Suga H, Kitabatake A, Sagawa K. End systolic pressure determines stroke volume from fixed end diastolic volume in the isolated canine left ventricle, under a constant contractile state. Circ Res 1979; 44: 238.

[14] Campbell KB, Ringo JA. Use of elastance-resistance models to characterize natural ejecting left ventricle. Proceeding of 6th International Conference and Workshop, Cardiovascular Dynamics Society, Philadelphia 1984.

[15] Campbell KB, Ringo JA, Knowlen GG, Kirkpatrick RD, Schmidt SL. Validation of optional elastance-resistance left ventricle pump model. Am J Physiol 1986; 251: H382.

[16] Shroff SG, Janicki JS, Weber KT. Left ventricular systolic dynamics in terms of its chamber mechanical properties. Am J Physiol 1983; 245: H110.

[17] Shroff SG, Janicki JS, Weber KT. Evidence and quatitation of left ventricular systolic resistance. Am J Physiol 1985; 249: $\mathrm{H} 358$.

[18] Sheffer L, Santamore WP, Barnea O. Cardiovascular Simulation Toolbox. Cardiovasc Eng 2007; 7: 81-8. 